\title{
Careful Prescribing of Benzodiazepines during COVID-19 Pandemic: A Review
}

\author{
Ruchita Agrawal $1,2 *$ \\ 'Seven Counties Services, Louisville KY, USA (Formerly, Centerstone) \\ ${ }^{2}$ Department of Psychiatry, University of Louisville, Louisville KY, USA
}

Article Info

\section{Article Notes}

Received: August 8, 2020

Accepted: October 12, 2020

\section{*Correspondence:}

Dr. Ruchita Agrawal MD, FAPA, Associate Chief Medical officer Adult services, Seven Counties Services, 708 Magazine street, Louisville KY 40204 USA; Telephone No: 5025898926; Email: ragrawal@sevencounties.org.

C) 2020 Agrawal R. This article is distributed under the terms of the Creative Commons Attribution 4.0 International License.

\section{Keywords:}

Benzodiazepine

Addiction

Over-dose

Anxiety

Insomnia

COVID- 19 pandemic

\section{Abstract}

Benzodiazepines have been commonly prescribed for the treatment of anxiety and insomnia in the last few decades. There has been a rising concern regarding safety of benzodiazepines due to overdose related deaths, addictions, and cognitive side effects. COVID- 19 pandemic is expected to cause a mental health crisis. Several studies have shown an increase in anxiety and insomnia. This could mean that prescriptions of benzodiazepine could increase due to increase in anxiety and insomnia. We caution health care providers to use best practices and treat patients with psychotherapy as the first line of treatment and not pharmacotherapy. Prescription Drug Monitoring programs (PDMPs) were started due to this concern of overdose deaths, diversion related to opioids and benzodiazepines. PDMPs are mandatory in most states in the United States of America now.We recommend all health care providers to look at their benzodiazepine prescribing practice, monitor PDMP data and make policies to implement changes in order to avoid the next crisis of benzodiazepines after opioids.

\section{Introduction}

Benzodiazepines have been around since the 1960s and they have been one of the most commonly prescribed medications worldwide. Over years, there has been a rising concern of the risk of overdose-related deaths, addiction, and long-term cognitive concerns with benzodiazepines. As per the Centers for Disease Control and Prevention (CDC), there were 67,367 deaths in the United States due to overdose in $2018^{1}$. Synthetic opioids were the main drugs involved in deaths. As per the National Institute on Drug Abuse (NIDA), benzodiazepines were involved in more than 30 percent of the opioid-related overdose-related deaths ${ }^{2}$. Due to these rising concerns, Food and Drug Administration (FDA) and CDC mandated changes. In 2016, the CDC came up with guidelines and recommended avoiding combined use when possible. Prescription drug monitoring program (PDMP) is an electronic way to monitor the dispensing of controlled substances used in most states in the United States of America (USA). Due to the concern of diversion of opioids, PDMPs started to flourish in 2002 and they are now mandated in most states in the USA. There have been several studies done on PDMP. Some studies have shown a decrease in the number of opioid and benzodiazepine prescriptions ${ }^{3}$. Others like the study done by Di Liang on Medicaid enrolled from 2010- 2017 did not find any change in benzodiazepine prescribing practice with mandating the use of PDMP4. 
There has been a staggering increase in the number of benzodiazepine prescriptions in the last couple of decades in the USA. According to a serial cross-sectional study (January 1, 2003, through December 31, 2015) done by S. Agarwal benzodiazepine prescriptions in outpatient settings increased substantially from $3.8 \%$ to $7.4 \%$ of visits $^{5}$. Psychiatrists prescribing benzodiazepines have been stable $(29.6 \%$ vs $30.2 \%)$ but primary care physicians have increased $(3.6 \% \text { vs } 7.5 \%)^{5}$. Primary care accounted for about half of all benzodiazepine prescriptions. This pattern of the rise of benzodiazepine prescriptions is very concerning amid the opioid epidemic and the COVID-19 pandemic.

As per NIDA, opioid-related overdose deaths increased from 3,442 in 1999 to 17,029 in 2017 but the number of deaths dropped to 14,975 from 2017-2018 in the USA. Deaths due to benzodiazepine overdose increased from 1,135 in 1999 to 11,537 in 2017 but it declined to 10,724 from 2017 and 2018 in the USA 2. As per the New York Times, this decrease was probably due to tightening of the opioid prescriptions ${ }^{6}$.

\section{COVID-19 and Mental Health}

The COVID-19 pandemic could cause a mental health crisis. Studies have shown an increase in anxiety and insomnia in health care providers as well as in the general population ${ }^{7-9}$. Per a survey done by the American Psychiatric Association in March 2020, 48 percent of the general population reported anxiety, 36 percent informed that pandemic has seriously affected their mental health and 19 percent reported insomnia 7. A systematic review and meta-analysis done by S.Pappa during this time of pandemic showed a prevalence of 23.2 percent anxiety and 22.8 percent depression in health care workers ${ }^{8}$. In one of the studies done in China on health care providers who treated COVID patients, 50.4 percent reported depression, 44.6 percent anxiety, 34 percent insomnia, 71.5 percent distress9. This rise in anxiety, insomnia, and depression is alarming in both the general population and health care workers. Hence, it can be anticipated that there will be an increase in use of benzodiazepine prescriptions.

Per the National Institue of Mental Health (NIMH), suicide was the second leading cause of death among individuals between the ages of 10 and 34, and the fourth leading cause of death among individuals between the ages of 35 and $54^{10}$. During the 20 years from 1999 through 2018, the total suicide rate increased $35 \%$ from 10.5 to 14.2 per 100,000 10. Poverty, unemployment, opioid use, are all risk factors for suicides. There has been an upswing in unemployment due to the COVID-19 pandemic, so there could be an increase in suicides as well. Overdosing is one of the common methods of the suicide attempt. Amid this pandemic, we need to be very careful about what we are prescribing to avoid intentional and accidental overdose.
Social isolation and lack of ready access to support is another risk factor ${ }^{11}$. Social isolation measures taken due to pandemic could have a ripple effect on mental health as the support network could be disrupted for some people. There is a lot of apprehension about getting sick, dying, finances, housing, unemployment, childcare due to pandemic. The longer this pandemic continues the worse it is going to be for mental health. Stress can activate the hypothalamicpituitary axis which indeed increases cortisol. Cortisol is a steroid hormone that plays a role in stress response and immunity along with other functions. Chronic stress could increase inflammatory conditions in the body, decrease immunity ${ }^{12}$. During this time of pandemic when we don't have any treatment for COVID-19, we need our immunity to be good to fight this infection.

Insomnia can be triggered by acute stress and over time it can become chronic. People who have high sleep reactivity are more prone to insomnia with stress. Sleep reactivity is a trait that some individuals can have, it is also linked to the risk of depression and anxiety ${ }^{13}$. There has been an increase in insomnia due to the pandemic and it could be explained due to the cognitive distortions, ruminations that some people can have at bedtime. Ruminations in bed can increase anxiety and relate bedtime with anxiety, therefore relaxation techniques like progressive muscle relaxation can help us relax at bedtime and decrease sleep latency. A study done by Palagini on subjects with insomnia showed low resilience was related to high stress-related sleep reactivity, emotional dysregulation, and hyperarousal ${ }^{14}$.

American College of Physicians released guidelines for the management of insomnia in 2016. According to those guidelines, Cognitive Behavioral Therapy for insomnia (CBT- I) is the recommended first treatment modality. If CBT-I alone is ineffective then short-term use of sedating medications is recommended after discussing the risk, benefits, and making shared decisions ${ }^{15}$. CBT-I includes various techniques like sleep hygiene to regulate the circadian rhythm, matching sleep opportunity with sleep efficiency, restoring homeostatic regulation of sleep by sleep restriction, relaxation techniques, and stimulus control. A systematic review done to compare medications with CBT-I showed CBT-I to be more effective in the long term due to more durable benefits and fewer side effects ${ }^{16}$.

Benzodiazepine use has been associated with cognitive side effects, so there have been concerns about the risk of dementia with long term use. Benzodiazepines act as GABA-A receptor agonists, they allosterically modulate GABA-A receptors. The inhibitory GABA-A could modulate the cognition and cause negative cognitive effects. These receptors are located not just in the limbic system but in the cerebellum, spinal cord, cerebral cortex as well. There have been several studies and meta-analyses done over the past 2 decades. A meta-analysis was done 
by Barker, Greenwood, and Jackson $(2004,2005)$ that showed long-term cognitive side effects ${ }^{17}$. S. Crowe did a meta-analysis and published in 2017 , the meta-analysis revealed statistically significant, negative effects for the cognitive domains of working memory, processing speed, divided attention, visuoconstruction, recent memory, and expressive language ${ }^{18}$. Billioti de Gage and colleagues did a study in Canada on the link between benzodiazepines and Alzheimer's dementia and they found out a two-fold increase in risk with more than 180 days of use. They also found out that long-acting benzodiazepines increased the risk of more than shorter-acting benzodiazepines ${ }^{19}$. A nationwide large cohort and case-control study done on Danish patients published in 2020 did not reveal an association between benzodiazepine and z-drugs and subsequent dementia ${ }^{20}$.

\section{Discussion}

There is a lot of debate on the use of benzodiazepines in the medical community. Some support the use due to immediate benefits and some don't due to addiction, tolerance, withdrawal, risk of cognitive decline, falls, etc. Conflicting studies makes it even harder to make the right decision at the time when patients are seen in the clinics. There are limited resources for psychotherapy and that makes it even harder for our primary care colleagues to treat anxiety and insomnia. Overall there is no denying the fact that we are seeing more overdose-related deaths, addiction problems, suicides ${ }^{2,10,21}$. Due to the COVID-19 pandemic, mental health crisis is expected and we are probably going to see even more rise in anxiety and insomnia. Benzodiazepines could be alluring during this time due to their "quick fix" nature, but we know due to this "quick fix", patients are not able to develop coping skills to cope with the stressors ${ }^{22-26}$. Patients get dependent on medicines and that's how the long-term use becomes an addiction problem. The first line of treatment for anxiety and insomnia is psychotherapy, so that should be used before moving on to pharmacotherapy. There are several types of psychotherapies available like Cognitive Behavioral Therapy (CBT) for anxiety, CBT for insomnia, supportive therapy, interpersonal therapy, trauma-focused therapy, etc. CBT can help develop rational thinking and help patients cope with their ruminations and worrying which indeed could help with anxiety and insomnia. Building a positive attitude by psychotherapy can last long and help us develop resilience to cope with any stressors in life. Resilience is a trait that shows how adaptable an individual is to stressful events. People with high resilience can adapt and are at low risk for anxiety, insomnia with stress. Whereas people with low resilience are at high risk for insomnia and anxiety during stressful events like the current pandemic. Low resilience could be the reason for the increase in the rates of anxiety and insomnia in some people with the current pandemic. Resilience could be internal or external. Internal resilience is inborn but external resilience could be achieved with the help of psychotherapy. Teaching this trait to people can help develop better-coping skills which could decrease the rates of anxiety and insomnia.

\section{Conclusion}

Current NIDA and CDC data of decrease in overdose deaths related to benzodiazepines and prescription opioids are reassuring. This shows that we can protect our community through careful prescribing practices. We recommend all health care providers to look at their benzodiazepine prescribing practice, monitor PDMP data, and make policies to implement changes to avoid the next crisis of benzodiazepines after opioids during this COVID-19 outbreak and thereafter.

\section{Author contribution}

RA is the project PI and wrote the manuscript. Author has approved the submission version of this manuscript.

\section{References}

1. https://www.cdc.gov/nchs/data/databriefs/db356-h.pdf. https:// www.cdc.gov/nchs/data/databriefs/db356-h.pdf. Accessed October 2, 2020.

2. Overdose Death Rates | National Institute on Drug Abuse (NIDA). https://www.drugabuse.gov/drug-topics/trends-statistics/ overdose-death-rates. Accessed October 2, 2020.

3. Strickler GK, Zhang K, Halpin JF, et al. Effects of mandatory prescription drug monitoring program (PDMP) use laws on prescriber registration and use and on risky prescribing. Drug Alcohol Depend. 2019; 199: 1-9. doi:10.1016/j.drugalcdep.2019.02.010

4. Liang D, Guo H, Shi Y. Mandatory use of prescription drug monitoring program and benzodiazepine prescribing among U.S. Medicaid enrollees. Subst Abus. November 2019; 1-8. doi:10.1080/08897077 .2019 .1686722

5. Agarwal SD, Landon BE. Patterns in outpatient benzodiazepine prescribing in the United States. JAMA Netw Open. 2019; 2(1): e187399. doi:10.1001/jamanetworkopen.2018.7399

6. Drug Overdose Deaths Drop in U.S. for First Time Since 1990 - The New York Times. https://www.nytimes.com/interactive/2019/07/17/ upshot/drug-overdose-deaths-fall.html. Accessed October 4, 2020.

7. New Poll: COVID-19 Impacting Mental Well-Being: Americans Feeling Anxious, Especially for Loved Ones; Older Adults are Less Anxious. https://www.psychiatry.org/newsroom/news-releases/new-pollcovid-19-impacting-mental-well-being-americans-feeling-anxiousespecially-for-loved-ones-older-adults-are-less-anxious. Accessed October 4, 2020.

8. Pappa S, Ntella V, Giannakas T, et al. Prevalence of depression, anxiety, and insomnia among healthcare workers during the COVID-19 pandemic: A systematic review and meta-analysis. Brain Behav Immun. 2020; 88: 901-907. doi:10.1016/j.bbi.2020.05.026

9. Lai J, Ma S, Wang Y, et al. Factors associated with mental health outcomes among health care workers exposed to coronavirus disease 2019. JAMA Netw Open. 2020; 3(3): e203976. doi:10.1001/ jamanetworkopen.2020.3976

10. NIMH » Suicide. https://www.nimh.nih.gov/health/statistics/ suicide.shtml. Accessed October 4, 2020. 
11. Baldessarini RJ. Epidemiology of suicide: recent developments. Epidemiol Psychiatr Sci. 2019; 29: e71. doi:10.1017/ S2045796019000672

12. Wolf JM, Nicholls E, Chen E. Chronic stress, salivary cortisol, and alpha-amylase in children with asthma and healthy children. Biol Psychol. 2008; 78(1): 20-28. doi:10.1016/j.biopsycho.2007.12.004

13. Kalmbach DA, Anderson JR, Drake CL. The impact of stress on sleep: Pathogenic sleep reactivity as a vulnerability to insomnia and circadian disorders. J Sleep Res. 2018; 27(6): e12710. doi:10.1111/ jsr.12710

14. Palagini L, Moretto U, Novi M, et al. Lack of Resilience Is Related to Stress-Related Sleep Reactivity, Hyperarousal, and Emotion Dysregulation in Insomnia Disorder. J Clin Sleep Med. 2018; 14(5): 759-766. doi:10.5664/jcsm.7100

15. Qaseem A, Wilt TJ, Weinberger SE, et al. Diagnosis and management of stable chronic obstructive pulmonary disease: a clinical practice guideline update from the American College of Physicians, American College of Chest Physicians, American Thoracic Society, and European Respiratory Society. Ann Intern Med. 2011; 155(3): 179-191. doi:10.7326/0003-4819-155-3-201108020-00008

16. Mitchell MD, Gehrman P, Perlis M, et al. Comparative effectiveness of cognitive behavioral therapy for insomnia: a systematic review. BMC Fam Pract. 2012; 13: 40. doi:10.1186/1471-2296-13-40

17. Barker MJ, Greenwood KM, Jackson M, et al. Cognitive effects of longterm benzodiazepine use: a meta-analysis. CNS Drugs. 2004; 18(1): 37-48. doi:10.2165/00023210-200418010-00004

18. Crowe SF, Stranks EK. The Residual Medium and Long-term Cognitive Effects of Benzodiazepine Use: An Updated Meta-analysis. Arch Clin Neuropsychol. 2018; 33(7): 901-911. doi:10.1093/arclin/acx120
19. Billioti de Gage S, Moride Y, Ducruet T, et al. Benzodiazepine use and risk of Alzheimer's disease: case-control study. BMJ. 2014; 349: g5205. doi:10.1136/bmj.g5205

20. Osler M, Jørgensen MB. Associations of Benzodiazepines, Z-Drugs, and Other Anxiolytics With Subsequent Dementia in Patients With Affective Disorders: A Nationwide Cohort and Nested Case-Control Study. Am J Psychiatry. 2020; 177(6): 497-505. doi:10.1176/appi. ajp.2019.19030315

21. Drug Overdose Mortality by State. https://www.cdc.gov/nchs/ pressroom/sosmap/drug_poisoning_mortality/drug_poisoning.htm. Accessed October 2, 2020.

22. Guina J, Merrill B. Benzodiazepines I: upping the care on downers: the evidence of risks, benefits and alternatives. J Clin Med. 2018; 7(2). doi:10.3390/jcm7020017

23. Gelpin E, Bonne 0, Peri T, et al. Treatment of recent trauma survivors with benzodiazepines: a prospective study. J Clin Psychiatry. 1996; 57(9): 390-394.

24. Mellman TA, Byers PM, Augenstein JS. Pilot evaluation of hypnotic medication during acute traumatic stress response. J Trauma Stress. 1998; 11(3): 563-569. doi:10.1023/A:1024460814230

25. Davydow DS, Gifford JM, Desai SV, et al. Posttraumatic stress disorder in general intensive care unit survivors: a systematic review. Gen Hosp Psychiatry. 2008; 30(5): 421-434. doi:10.1016/j. genhosppsych.2008.05.006

26. Agrawal R, Verma S, Halappanavar M. Measuring the effectiveness of benzodiazepine prescriptions control in community setting using prescription drug monitoring program (PDMP). Community Ment Health J. July 2020. doi:10.1007/s10597-020-00686-5 